\title{
Exclusive Window onto Higgs Yukawa Couplings
}

\author{
Alexander L. Kagan, ${ }^{1, *}$ Gilad Perez, ${ }^{2,3, \dagger}$ Frank Petriello, ${ }^{4,5, \sharp}$ Yotam Soreq, ${ }^{3, \S}$ Stoyan Stoynev, ${ }^{5, \uparrow}$ and Jure Zupan ${ }^{1, \|}$ \\ ${ }^{1}$ Department of Physics, University of Cincinnati, Cincinnati, Ohio 45221, USA \\ ${ }^{2}$ CERN Theory Division, CH-1211, Geneva 23, Switzerland \\ ${ }^{3}$ Department of Particle Physics and Astrophysics, Weizmann Institute of Science, Rehovot 7610001, Israel \\ ${ }^{4}$ High Energy Physics Division, Argonne National Laboratory, Argonne, Illinois 60439, USA \\ ${ }^{5}$ Department of Physics \& Astronomy, Northwestern University, Evanston, Illinois 60208, USA
}

(Received 18 June 2014; published 10 March 2015)

\begin{abstract}
We show that both flavor-conserving and flavor-violating Yukawa couplings of the Higgs boson to first- and second-generation quarks can be probed by measuring rare decays of the form $h \rightarrow M V$, where $M$ denotes a vector meson and $V$ indicates either $\gamma, W$ or $Z$. We calculate the branching ratios for these processes in both the standard model and its possible extensions. We discuss the experimental prospects for their observation. The possibility of accessing these Higgs couplings appears to be unique to the high-luminosity LHC and future hadron colliders, providing further motivation for those machines.
\end{abstract}

DOI: 10.1103/PhysRevLett.114.101802

PACS numbers: 14.80.Bn, 14.40.Be, 14.40.Nd

Introduction.-The discovery of a Higgs-like boson by the ATLAS and CMS collaborations [1,2] ushered in a new era of exploration driven by the desire to understand the properties of this new state. Current measurements only give information on the Higgs couplings to gauge bosons and third-generation fermions. In the well-measured decay modes, $h \rightarrow \gamma \gamma, W W$ and $Z Z$, they agree with the standard model (SM) values at the 20\%-30\% level [3,4].

In contrast, the couplings of the Higgs boson to the firstand second-generation fermions are only weakly constrained by the inclusive Higgs production cross sections. At the same time, they can receive large modifications in beyond-the-SM theories [5-7], making them interesting experimental targets. The ATLAS and CMS collaborations have studied the possibility of measuring the Higgs couplings to muons at the high-luminosity LHC (HLLHC) with encouraging results [8-10]. However, the question of whether the Higgs couplings to the other light fermions can be directly accessed is left completely open.

In this Letter we lay out a program to measure enhanced Higgs couplings to light quarks using the exclusive decays $h \rightarrow M V$, where $M$ denotes a vector meson and $V$ denotes either a $\gamma, W$ or $Z$. The possibility of using $h \rightarrow J / \psi \gamma$ to probe the Higgs coupling to charm quarks was recently pointed out in Ref. [11]. This coupling can also be accessed using charm-tagging techniques [7]. The modes studied here, on the other hand, allow access to Higgs couplings that are impossible to directly determine in other ways. For example, the $h \rightarrow \phi \gamma$ mode considered here allows direct access to the flavor-diagonal coupling of the Higgs boson

Published by the American Physical Society under the terms of the Creative Commons Attribution 3.0 License. Further distribution of this work must maintain attribution to the author(s) and the published article's title, journal citation, and DOI. to the strange quark. The $h \rightarrow \rho \gamma, \omega \gamma$ modes probe the Higgs couplings to up and down quarks, while the $h \rightarrow K^{* 0} \gamma, D^{* 0} \gamma, B^{* 0} \gamma, B_{s}^{* 0} \gamma$ modes probe the off-diagonal Yukawa couplings of the Higgs. We discuss the signatures of these rare radiative processes and also comment on $h \rightarrow M W, M Z$ decays, e.g., $h \rightarrow B^{(*)+} W^{-}$. Probes of the electroweak couplings of the Higgs boson via $h \rightarrow M Z$, $M W$ decays have been discussed in Ref. [12].

These rare decays are only accessible at the HL-LHC and future high-energy colliders, due to their small branching ratios (BRs). The predicted event rates at planned $e^{+} e^{-}$ facilities are too small, strengthening the motivation for future hadron colliders.

Theoretical framework.-We first consider the constraints on the Higgs Yukawa couplings coming from the inclusive Higgs production rate at the LHC. In our analysis, we assume that the Higgs boson is a singlet of the custodial symmetry preserved by electroweak symmetry breaking, with $C P$-conserving couplings and mass $m_{h} \simeq 125.7 \mathrm{GeV}$. The following phenomenological Lagrangian is used in our analysis:

$$
\begin{aligned}
\mathcal{L}_{\text {eff }}= & -\sum_{q=u, d, s} \bar{\kappa}_{q} \frac{m_{b}}{v} h \bar{q}_{L} q_{R}-\sum_{q \neq q^{\prime}} \bar{\kappa}_{q q^{\prime}} \frac{m_{b}}{v} h \bar{q}_{L} q_{R}^{\prime}+\text { H.c. } \\
& +\kappa_{Z} m_{Z}^{2} \frac{h}{v} Z_{\mu} Z^{\mu}+2 \kappa_{W} m_{W}^{2} \frac{h}{v} W_{\mu}^{+} W^{-\mu}
\end{aligned}
$$

where $v=246 \mathrm{GeV}$ is the Higgs vacuum expectation value. The $h \rightarrow \gamma \gamma$ amplitude can be written as

$$
M_{h \rightarrow \gamma \gamma}=\left\langle\gamma \gamma\left|\kappa_{\gamma} A_{\gamma} \frac{\alpha}{\pi} \frac{h}{v} F^{\mu \nu} F_{\mu \nu}\right| h\right\rangle,
$$

where at one-loop $A_{\gamma} \approx-0.81$ in the SM [13]. The custodial symmetry implies $\kappa_{W}=\kappa_{Z}=\kappa_{V}$, and $C P$ conservation 
implies real $\bar{\kappa}_{q}, \kappa_{V, \gamma}$, and $\bar{\kappa}_{q q^{\prime}}=\bar{\kappa}_{q^{\prime} q}^{*}$. Note that $\bar{\kappa}_{q}$ and $\bar{\kappa}_{q q^{\prime}}$ are normalized to the SM $b$-quark Yukawa coupling. The SM limit corresponds to $\kappa_{\gamma}=\kappa_{V}=1$, and $\bar{\kappa}_{s}=m_{s} / m_{b} \simeq 0.020$, $\bar{\kappa}_{d}=m_{d} / m_{b} \simeq 1.0 \times 10^{-3}, \quad \bar{\kappa}_{u}=m_{u} / m_{b} \simeq 4.7 \times 10^{-4}$. The quark masses are evaluated at $\mu=m_{h}$ using NNLO running in the $\bar{M} S$ scheme with low-energy inputs from Ref. [14]. The $\bar{\kappa}_{q q^{\prime}}$ vanish in the SM. Any deviations from these relations would signal the presence of new physics.

Constraints from the current data.-In Ref. [7] the LHC inclusive production rate was used to place an indirect bound on the charm Yukawa coupling. Here, we adapt this analysis to the other Yukawa couplings, $\bar{\kappa}_{i}$. The current ATLAS [15], CMS [16], and Tevatron [17] Higgs measurements are included (based on Tables 13 and 14 of Ref. [18]), as are the indirect constraints from the LEP electroweak precision measurements [19]. For simplicity, correlations between the different measurements are neglected and asymmetric uncertainties are symmetrized. The quark antiquark Higgs-fusion cross section is evaluated at next-toleading order in $\alpha_{s}$ based on the bottom fusion cross section obtained in Ref. [20] using MSTW parton distribution functions [21]. Below, we check that our fit results are stable against uncalculated higher-order corrections by varying our production cross sections by $40 \%$, the estimated theoretical error at next-to-leading order [20]. The resulting shifts in the bounds on the $\bar{\kappa}_{i}$ are extremely small.

We begin with the flavor-conserving couplings. A naive $\chi^{2}$ fit to the data that fixes all Higgs couplings to their SM values, except for one of the up, down, or strange Yukawa couplings at a time, leads to the $95 \%$ confidence level (C.L.) bounds

$$
\left|\bar{\kappa}_{u}\right|<1.0, \quad\left|\bar{\kappa}_{d}\right|<0.9, \quad\left|\bar{\kappa}_{s}\right|<0.7 .
$$

If all of the Higgs couplings (including $h \rightarrow W W$, $Z Z, \gamma \gamma, g g, Z \gamma, b \bar{b}$ and $\tau \bar{\tau}$ ) are allowed to vary from their SM values, we get the weaker $95 \%$ C.L. bounds

$$
\left|\bar{\kappa}_{u}\right|<1.3, \quad\left|\bar{\kappa}_{d}\right|<1.4, \quad\left|\bar{\kappa}_{s}\right|<1.4 .
$$

The 95\% C.L. upper bounds obtained for the offdiagonal couplings, when modifying only a single Yukawa coupling at a time (or allowing for modification of the other Higgs couplings as above), are

$$
\left|\bar{\kappa}_{q q^{\prime}}\right|<0.6(1),
$$

for $q, q^{\prime} \in u, d, s, c, b$ and $q \neq q^{\prime}$. The bounds are $10 \%-$ $20 \%$ stronger for couplings only involving sea quarks, as their slightly smaller direct production cross section does not compensate for the increased decay width.

Inclusive Higgs rate measurements cannot distinguish between the individual $\bar{\kappa}_{q q^{\prime}}$. The weakest indirect bound from low-energy observables is found to be $\left|\bar{\kappa}_{b s}\right|<8 \times 10^{-2}$ [22] (see also Refs. [23,24]). However, such bounds are model dependent. For instance, if the Higgs boson is part of

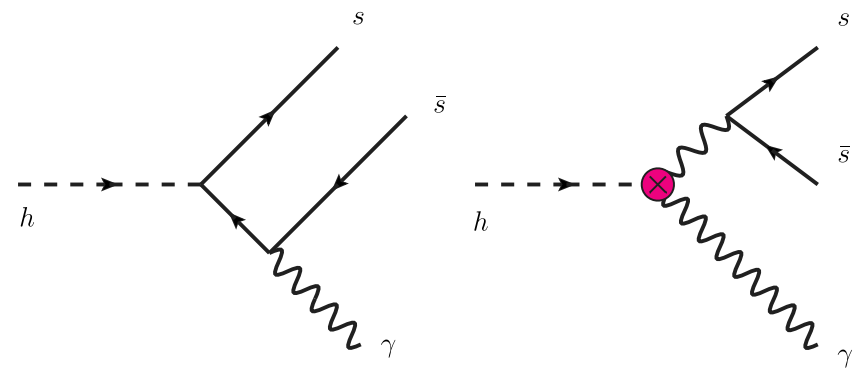

FIG. 1 (color online). Direct-amplitude diagram (left) and indirect-amplitude diagram (right) contributing to $h \rightarrow \phi \gamma$.

a multiplet that approximately conserves the flavor symmetries, its contributions could be (partially) canceled by other members of the multiplet. The latter could mostly decay to light quarks or have reduced production rates, thus remaining unobserved.

Flavor-conserving photonic decays.-We begin with $h \rightarrow \phi \gamma$. The decay amplitude receives two dominant contributions, which we denote as direct and indirect; see Fig. 1. The indirect contribution proceeds through the $h \gamma \gamma$ coupling, followed by the fragmentation of $\gamma^{*} \rightarrow \phi$. In our analysis, we use the on-shell $h \rightarrow \gamma \gamma$ amplitude (2). The error due to this is small, $\mathcal{O}\left(m_{\phi}^{2} / m_{h}^{2}\right)$. Similarly, the indirect contribution from $h \rightarrow \gamma Z$ is neglected, because it is suppressed by the off-shell $Z$. The direct amplitude involves a hard $h \rightarrow s \bar{s} \gamma$ vertex, where an intermediate $s$-quark line with an off-shellness $Q^{2} \sim \mathcal{O}\left(m_{h}^{2}\right)$ is integrated out. Its evaluation is a straightforward application of QCD factorization [25]. The largest sensitivity to the Higgsstrange quark coupling is due to the interference of the two amplitudes. (The direct amplitude by itself yields $\mathrm{BR}_{h \rightarrow \phi \gamma} \sim 10^{-11}$ in the SM.) However, the interference only involves the real part of the coupling, $\operatorname{Re}\left(\bar{\kappa}_{s}\right)$. Working in the limit of real $\bar{\kappa}_{s}$, the $h \rightarrow \phi \gamma$ decay amplitude is

$$
M_{s s}^{\phi}=\frac{Q_{s} e}{2} \epsilon^{\phi} \times \epsilon^{\gamma}\left(\bar{\kappa}_{s} \frac{m_{b}}{v} f_{\perp}^{\phi}\langle 1 / u \bar{u}\rangle_{\perp}^{\phi}+\frac{4 \alpha}{\pi v} \kappa_{\gamma} A_{\gamma} \frac{f_{\phi} m_{h}^{2}}{m_{\phi}}\right),
$$

where the first and second terms are the direct and indirect contributions; $f_{\perp}^{\phi}$ and $\langle 1 / u \bar{u}\rangle_{\perp}^{\phi}$ are the decay constant and inverse moment of the light-cone distribution amplitude (LCDA) defined in Eq. (8), $Q_{s} e=-e / 3$ is the strange quark electric charge, and $\varepsilon_{\gamma}$ and $\varepsilon_{\phi}$ are the $\gamma$ and $\phi$ polarization vectors. We have used the definition $\left\langle\phi\left|J_{\mathrm{EM}}^{\mu}(0)\right| 0\right\rangle=f_{\phi} m_{\phi} \epsilon_{\phi}^{\mu}$ for the $\phi$ decay constant $f_{\phi}$, where $J_{\mathrm{EM}}^{\mu}=\sum_{f} Q_{f} \bar{f} \gamma^{\mu} f$ is the electromagnetic current. Note that for $C P$-violating couplings $M_{S S}^{\phi}$ is sensitive to the phase between $A_{\gamma}$ and $\bar{\kappa}_{\gamma}$.

The LCDA convolution integral is

$$
\langle 1 / u \bar{u}\rangle_{\perp}^{\phi}=\int_{0}^{1} d u \frac{\phi_{\perp}^{\phi}(u)}{u(1-u)} .
$$


The leading twist chiral-odd LCDA $\phi_{\perp}(u)$ is defined through the following matrix element of the transversely polarized $\phi$ meson on the light-cone [26,27]:

$$
\begin{aligned}
& \left\langle\phi\left(p, \varepsilon_{\perp}\right)\left|\bar{s}(x) \sigma_{\mu \nu} s(0)\right| 0\right\rangle \\
& \quad=-i f_{\perp}^{\phi} \int_{0}^{1} d u e^{i u p \times x}\left(\varepsilon_{\perp \mu} p_{\nu}-\varepsilon_{\perp \nu} p_{\mu}\right) \phi_{\perp}^{\phi}(u) .
\end{aligned}
$$

The partial decay width for $h \rightarrow \phi \gamma$ is

$$
\Gamma_{h \rightarrow \phi \gamma}=\frac{1}{8 \pi} \frac{1}{m_{h}}\left|M_{s s}^{\phi}\right|^{2}
$$

where $\left|\epsilon_{\perp}^{\phi} \times \epsilon^{\gamma}\right|=1$ for the two possible photon polarizations so that the two corresponding decay amplitudes are equal in size. The decay widths for $h \rightarrow \rho \gamma$ and $h \rightarrow \omega \gamma$ are similarly given by

$$
\Gamma_{h \rightarrow \rho \gamma}=\frac{\left|M_{d d}^{\rho}-M_{u u}^{\rho}\right|^{2}}{16 \pi m_{h}}, \quad \Gamma_{h \rightarrow \omega \gamma}=\frac{\left|M_{d d}^{\omega}+M_{u u}^{\omega}\right|^{2}}{16 \pi m_{h}},
$$

where the amplitudes are obtained from $M_{s s}^{\phi}$ via the replacements $s \rightarrow u, d$ and $\phi \rightarrow \rho, \omega$. For simplicity, we have neglected $\omega-\phi$ mixing.

In our numerical estimates the Gegenbauer polynomial expansions of the $\phi_{\perp}$ are truncated at second order, yielding $\langle 1 / u \bar{u}\rangle_{\perp}^{\phi}=6.84(42),\langle 1 / u \bar{u}\rangle_{\perp}^{\rho}=6.84(36)$, and $\langle 1 / u \bar{u}\rangle_{\perp}^{\omega}=$ 6.84(72), using the inputs from Ref. [28] and fixing $\mu=1 \mathrm{GeV}$. The decay constants are $f_{\phi}=0.235(5) \mathrm{GeV}$, $f_{\rho}=0.216(6) \mathrm{GeV}$, and $f_{\omega}=0.187(10) \mathrm{GeV}$ [28]. We estimate the error on our LO calculation by varying the renormalization scale for $f_{\perp}^{\phi, \rho, \omega}$ in the range $[0.5,10] \mathrm{GeV}$. The variation is combined in quadrature with the errors quoted in Ref. [28] to obtain $f_{\perp}^{\phi}=0.191(28) \mathrm{GeV}$, $f_{\perp}^{\rho}=0.160(25) \mathrm{GeV}, f_{\perp}^{\omega}=0.139(27) \mathrm{GeV}$. Normalizing to the $h \rightarrow b \bar{b}$ branching ratio gives

$$
\begin{aligned}
& \frac{\mathrm{BR}_{h \rightarrow \phi \gamma}}{\mathrm{BR}_{h \rightarrow b \bar{b}}}=\frac{\kappa_{\gamma}\left[(3.0 \pm 0.3) \kappa_{\gamma}-0.78 \bar{\kappa}_{s}\right] \times 10^{-6}}{0.57 \bar{\kappa}_{b}^{2}}, \\
& \frac{\mathrm{BR}_{h \rightarrow \rho \gamma}}{\mathrm{BR}_{h \rightarrow b \bar{b}}}=\frac{\kappa_{\gamma}\left[(1.9 \pm 0.2) \kappa_{\gamma}-0.24 \bar{\kappa}_{u}-0.12 \bar{\kappa}_{d}\right] \times 10^{-5}}{0.57 \bar{\kappa}_{b}^{2}}, \\
& \frac{\mathrm{BR}_{h \rightarrow \omega \gamma}}{\mathrm{BR}_{h \rightarrow b \bar{b}}}=\frac{\kappa_{\gamma}\left[(1.6 \pm 0.2) \kappa_{\gamma}-0.59 \bar{\kappa}_{u}-0.29 \bar{\kappa}_{d}\right] \times 10^{-6}}{0.57 \bar{\kappa}_{b}^{2}},
\end{aligned}
$$

where we have neglected the smaller $\bar{\kappa}_{s, d, u}^{2}$ terms. When calculating the theoretical error on the indirect amplitude, we have added in quadrature an additional uncertainty associated with the scale choice of the electromagnetic coupling that appears. The $\mathrm{SM} \mathrm{BR}_{h \rightarrow b \bar{b}}=0.57$ is kept explicit in the denominators. The numerators thus give the $h \rightarrow(\phi, \rho, \omega) \gamma$ branching ratios if the Higgs boson has the SM total decay width. The expected deviation from the SM

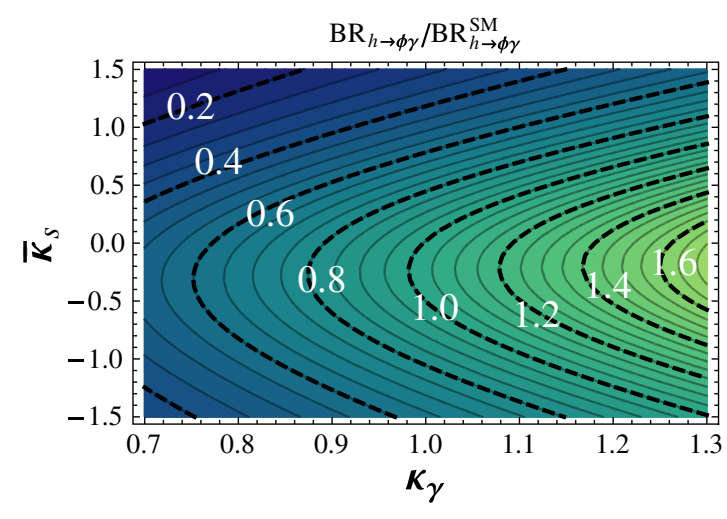

FIG. 2 (color online). The expected deviation in the branching ratio $h \rightarrow \phi \gamma$ relative to its $\mathrm{SM}$ value as a function of $\kappa_{\gamma}$ and $\bar{\kappa}_{s}$.

$h \rightarrow \phi \gamma$ branching ratio is shown in Fig. 2, as a function of $\kappa_{\gamma}$ and $\bar{\kappa}_{s}$.

The coefficients multiplying $\bar{\kappa}_{s, d, u}$ in Eq. (11) have a relative error of $\mathcal{O}(20 \%)$. This means that for $\bar{\kappa}_{i} \sim \mathcal{O}(1)$, deviations from the SM predictions for $h \rightarrow(\phi, \rho, \omega) \gamma$ can be significantly greater than the SM errors. The latter can be systematically reduced through advances in lattice QCD and measurements of the leptonic $\phi, \rho$, and $\omega$ decays. The BR predictions are relatively insensitive to other nonperturbative QCD effects, e.g., power corrections. For instance, $h \rightarrow g g \rightarrow g \bar{q} q \gamma$ yields a higher Fock state contribution to $h \rightarrow \phi \gamma$ of $\mathcal{O}(\mathrm{few}) \times 10^{-4}$ of the SM BR. Finally, the electroweak amplitude due to a $W$ or $Z$ and a $c$ or $s$ quark in the loop (the Higgs boson attaches to the gauge boson) scales as $\mathcal{O}\left(\alpha_{W} / 4 \pi\right) \times \mathcal{O}\left(m_{\phi} / m_{h}\right)$, yielding $O\left(10^{-2}\right)$ of the SM $h \rightarrow \phi \gamma$ branching ratio.

Flavor-violating photonic decays.-The radiative decays $h \rightarrow V \gamma$, where $V=B_{s}^{* 0}, B_{d}^{* 0}, K^{* 0}, D^{* 0}$ provide interesting possibilities to probe the flavor-violating Higgs couplings $\bar{\kappa}_{b s, s b}, \bar{\kappa}_{b d, d b}, \bar{\kappa}_{s d, d s}$, and $\bar{\kappa}_{c u, u c}$. They only receive direct amplitude contributions, since photon splitting preserves flavor. The $h \rightarrow K^{* 0} \gamma$ rate is readily obtained from the results of the previous section, yielding an $\mathcal{O}\left(10^{-8}\right)$ branching ratio for $\bar{\kappa}_{d s} \sim \mathcal{O}(1)$, out of reach of planned colliders. We thus focus on the decays to heavy mesons.

The essential difference with respect to the light mesons is that the $B_{(s)}^{* 0}$ and $D^{* 0}$ LCDA are heavily weighted toward the $b$ and $c$ quarks (we treat the $c$ quark as heavy, $\left.m_{c} \gg \Lambda_{\mathrm{QCD}}\right)$. For $h \rightarrow \bar{B}_{s}^{* 0} \gamma$, the dominant leading power contribution, in a general $R_{\xi}$ gauge, due to photon emission from the intermediate $s$ quark, is

$\Gamma_{h \rightarrow \bar{B}_{s}^{* 0} \gamma}=\frac{1}{8 \pi} \frac{1}{m_{h}}\left(\frac{f_{B_{s}} m_{B_{s}}}{2} \frac{m_{b}}{v} \frac{Q_{s} e_{0}}{\lambda_{B}(\mu)}\right)^{2} \frac{\left|\bar{\kappa}_{b s}\right|^{2}+\left|\bar{\kappa}_{s b}\right|^{2}}{2}$.

heavy quark effective theory sum rule estimates of the inverse moment of the $B$ meson LCDA yield $\lambda_{B}(\mu)=$ (460 \pm 110$) \mathrm{MeV}$ for $\mu=1 \mathrm{GeV}$ [29] (see also Ref. [30]). Note that $\lambda_{B}$ can be determined from $B \rightarrow \ell \nu \gamma$. Present limits, including NLO radiative corrections, yield a result 
compatible with the above estimate [31,32]. We have assumed flavor $\mathrm{SU}(3)$ symmetry so that $\lambda_{B}$ is the same for $B_{s}^{0}$ and $B_{d}^{0}$. Numerically, one has

$$
\frac{\mathrm{BR}_{h \rightarrow \bar{B}_{s}^{* 0} \gamma}}{\mathrm{BR}_{h \rightarrow b \bar{b}}}=\frac{\mathrm{BR}_{\bar{B}_{s}^{*} \gamma}^{(1)} \gamma}{0.57 \bar{\kappa}_{b}^{2}} \frac{\left|\bar{\kappa}_{b s}\right|^{2}+\left|\bar{\kappa}_{s b}\right|^{2}}{2},
$$

where $\operatorname{BR}_{\bar{B}_{s}^{* 0} \gamma}^{(1)}=(2.1 \pm 1.0) \times 10^{-7}$. The $h \rightarrow \bar{B}^{* 0} \gamma$ and $h \rightarrow D^{* 0} \gamma$ branching ratios are obtained by replacing $\bar{\kappa}_{b s, s b}$ with $\bar{\kappa}_{b d, d b}$ and $\bar{\kappa}_{c u, u c}$, respectively, with $\mathrm{BR}_{\bar{B}^{* 0} \gamma}^{(1)}=$ $(1.4 \pm 0.7) \times 10^{-7}$ and $\mathrm{BR}_{D^{* 0 \gamma}}^{(1)}=(8.6 \pm 8.3) \times 10^{-8}$. We have taken $\lambda_{D}=\lambda_{B}$ but have inflated the errors on $\lambda_{D}$ by a factor of 2. For the decay constants, we have used the FLAG averages $f_{B_{s}}=228(5) \mathrm{MeV}, f_{B}=191(4) \mathrm{MeV}$, and $f_{D}=209(3) \mathrm{MeV}$ [33].

The radiative decays to $B^{* 0}$ and $B_{s}^{* 0}$ are negligible in the $\mathrm{SM}$, where $\bar{\kappa}_{i j}=0$. Thus, their observation at future highluminosity hadron colliders would provide definitive evidence for new physics in the Higgs Yukawa sector.

Exclusive decays with $W$ and Z.-The charged $h \rightarrow$ $M^{-} W^{+}$decays differ qualitatively from the radiative decays. The $W$ attaches itself to a charged current, allowing probes of flavor-violating Higgs - top quark couplings. The complication is that the $W$ can have both transverse and longitudinal polarizations, yielding lengthier analytical expressions to be presented elsewhere [34]. For the most promising mode, we find

$$
\frac{\mathrm{BR}_{h \rightarrow B^{*-} W^{+}}}{\mathrm{BR}_{h \rightarrow b \bar{b}}} \simeq \frac{1.2 \times 10^{-10}\left[\kappa_{V}^{2}+22 \bar{\kappa}_{t u}^{2}+26 \bar{\kappa}_{u t}^{2}+\cdots\right]}{0.57 \bar{\kappa}_{b}^{2}},
$$

where only the potentially largest contributions are shown. The bounds on $\bar{\kappa}_{t u, u t}$ and $\bar{\kappa}_{t c, c t}$ from $t \rightarrow h u, h c$ decays are $[35,36]\left(\left|\bar{\kappa}_{t c}\right|^{2}+\left|\bar{\kappa}_{c t}\right|^{2}+\left|\bar{\kappa}_{t u}\right|^{2}+\left|\bar{\kappa}_{u t}^{2}\right|\right)^{1 / 2}<7.1$, which implies that $\mathrm{BR}_{h \rightarrow B^{*-} W^{+}} \leq 1.6 \times 10^{-7}$ is allowed.

The $h \rightarrow M Z$ decays feature smaller interference between the direct and indirect amplitudes and are less useful for measuring the Higgs couplings to light quarks.

Future experimental perspectives.-We begin by estimating the number of events at future collider facilities. We focus on the $h \rightarrow \phi \gamma$ mode and use PYTHIA 8.1 [37] to estimate its main features in proton-proton collisions at the LHC with the center of-mass energy of $14 \mathrm{TeV}$. The main $\phi$ decay modes $\left(K_{L, S}, K^{ \pm}, \pi^{ \pm}\right.$, and $\left.\pi^{0}\right)$ were explored. In $\approx 75 \%$ of the decays the kaons or pions and the prompt photon have $|\eta|<2.4$ and are thus within the minimal fiducial volume of the ATLAS and CMS experiments. We therefore adopt the geometrical acceptance factor of $A_{g}=0.75$ below but do not include other efficiency or trigger factors.

We focus on three facilities that were considered by the Snowmass Higgs working group [10]: the HL-LHC, a high-energy LHC (HE-LHC), and a VLHC. The Higgs production cross sections at these machines are obtained from the LHC Higgs cross section working group [38]. We have assumed two detectors for the HL-LHC and a single detector for the other colliders.

We estimate the reach in $\bar{\kappa}_{s}$ that can be obtained, given the current theoretical uncertainties and the expected statistical errors. For simplicity, we assume $\kappa_{\gamma}=1$ as in the SM. The significance of a deviation in the measured value of $\mathrm{BR}_{h \rightarrow \phi \gamma}$ with respect to its $\mathrm{SM}$ prediction can be quantified by $\mathcal{S}=\left|\mathrm{BR}_{h \rightarrow \phi \gamma}-\mathrm{BR}_{h \rightarrow \phi \gamma}^{\mathrm{SM}}\right| /\left(\delta \mathrm{BR}_{h \rightarrow \phi \gamma}\right)$, where $\left(\delta \mathrm{BR}_{h \rightarrow \phi \gamma}\right)^{2}=$ $\mathrm{BR}_{h \rightarrow \phi \gamma} /\left(\sigma_{h} \mathcal{L} A_{\mathrm{g}}\right)+\left(\delta \mathrm{BR}_{h \rightarrow \phi \gamma}^{\text {th }}\right)^{2}$ is the estimated uncertainty. The first term is the statistical uncertainty $\left(\sigma_{h}\right.$ is the total Higgs production cross section and $\mathcal{L}$ is the integrated luminosity), while the second term is the theoretical one, $\delta \mathrm{BR}_{h \rightarrow \phi \gamma}^{\text {th }} \approx 0.3 \times 10^{-6}$ for $\kappa_{\gamma}=1$; see Eq. (11). Our criterion for a large-enough deviation from the SM prediction is $\mathcal{S} \geq 3$. Our results are summarized in Table I. Only a few events are expected in future electron-positron colliders (ILC, CLIC, TLEP). Thus, the possibility of observing this mode appears to be unique to the hadron machines.

The $h \rightarrow \phi \gamma$ mode offers several promising experimental handles. The decay products, kaons or pions, fly in a narrow cone $\Delta R<0.1$, with tens of $\mathrm{GeV}$ of energy. They reach the detector before they decay (except the $K_{S}$ and $\pi^{0}$, which have much shorter lifetimes). The most apparent features for identification of the charged decay modes are the near collinearity of the photon and the $\phi$ jet in the transverse plane, the jet substructure information (two close high- $p_{T}$ tracks in a narrow cone), and the ditrack invariant mass distribution assuming kaons or pions. A detailed experimental simulation will be required to determine if this signature is feasible.

The $h \rightarrow \rho \gamma$ and $h \rightarrow \omega \gamma$ modes have rates comparable to or larger than the $\phi$ channel; see Eq. (11). The $\rho$ decays almost exclusively to $\pi^{+} \pi^{-}$. This is a relatively clean mode, similar to $\phi \rightarrow K^{+} K^{-}$, featuring two tracks with high transverse momenta and a proper invariant mass. The $\omega$ decays to $\pi^{+} \pi^{-} \pi^{0}$. This will be harder to trigger on than the $\rho$ or $\phi$ modes, as the transverse momenta of the charged pions are lower and the hard-to-identify neutral pion smears the observable quantities. A detailed experimental study is required to assess the feasibility of this channel. The $h \rightarrow$ $\bar{B}^{* 0} \gamma$ mode is more difficult, as the $B^{* 0}$ decays to $B^{0} \gamma$, leading to a $b$-jet $+\gamma$ final state. More study of this mode is needed.

TABLE I. Three future hadron colliders with expected centerof-mass energies, integrated luminosities, number of $h \rightarrow \phi \gamma$ events for $\bar{\kappa}_{s}=\bar{\kappa}_{s}^{\mathrm{SM}}=m_{s} / m_{b}$, the minimal (maximal) values of $\bar{\kappa}_{s}$ that can be probed with present (fourth column) and negligible (last column) theory error; see text.

\begin{tabular}{lcrcc}
\hline \hline$\sqrt{5}[\mathrm{TeV}]$ & $\int \mathcal{L} d t\left[\mathrm{fb}^{-1}\right]$ & $\begin{array}{c}\text { No. of events } \\
(\mathrm{SM})\end{array}$ & $\bar{\kappa}_{s}>(<)$ & $\bar{\kappa}_{s}^{\text {stat }}>(<)$ \\
\hline 14 & 3000 & 770 & $0.56(-1.2)$ & $0.27(-0.81)$ \\
33 & 3000 & 1380 & $0.54(-1.2)$ & $0.22(-0.75)$ \\
100 & 3000 & 5920 & $0.54(-1.2)$ & $0.13(-0.63)$ \\
\hline \hline
\end{tabular}


Conclusions.-In this Letter, we have shown that rare Higgs boson decays to vector mesons can explore the structure of the Higgs Yukawa couplings to the first- and second-generation quarks. Directly accessing the couplings of the Higgs boson to the lightest quarks was previously thought to be impossible. Rare decays of the form $h \rightarrow M V$ offer sensitivity to both flavor-conserving and flavorviolating couplings of the Higgs boson. They are theoretically calculable, experimentally promising, and should become a priority at the LHC Run II and at future hadron colliders. We look forward to further investigation of these ideas.

We thank the CERN theory group, where this work was initiated, for its hospitality. A. L. K. and J. Z. thank M. Neubert, and F. P. thanks K. Mishra for discussions. The work of A. L. K. is supported by DOE Grant No. DE-SC0011784. G. P. is supported by the Minerva foundation, the IRG, ERC, and by the Gruber award. F. P. is supported by DOE Grants No. DE-FG02-91ER40684 and No. DE-AC02-06CH11357. S. S. is supported by DOE Grant No. DE-FG02-91ER40684. J.Z. is supported in part by the U.S. National Science Foundation under CAREER Grant No. PHY-1151392.

*kaganal@ucmail.uc.edu

† gilad.perez@cern.ch

*f-petriello@northwestern.edu

§yotam.soreq@weizmann.ac.il

stoyan.stoynev@cern.ch

"zupanje@ucmail.uc.edu

[1] G. Aad et al. (ATLAS Collaboration), Phys. Lett. B 716, 1 (2012).

[2] S. Chatrchyan et al. (CMS Collaboration), Phys. Lett. B 716, 30 (2012).

[3] (ATLAS Collaboration), Report No. ATLAS-CONF-2013-034.

[4] (CMS Collaboration), Report No. CMS-PAS-HIG-13-005.

[5] See, e.g., models that lead to large deviations from the SM light quark Yukawa couplings: G. F. Giudice and O. Lebedev, Phys. Lett. B 665, 79 (2008); C. Delaunay, C. Grojean, and G. Perez, J. High Energy Phys. 09 (2013) 090.

[6] It is not inconceivable that light quark masses are not induced by the Higgs VEV, but by other subdominant sources of electroweak symmetry breaking.

[7] C. Delaunay, T. Golling, G. Perez, and Y. Soreq, Phys. Rev. D 89, 033014 (2014).

[8] (ATLAS Collaboration), Report No. ATLAS-CONF-2013-010.

[9] (CMS Collaboration), Report No. CMS PAS HIG-13-007.

[10] S. Dawson, A. Gritsan, H. Logan, J. Qian, C. Tully, R. Van Kooten, A. Ajaib, and A. Anastassov et al., arXiv:1310.8361.

[11] G. T. Bodwin, F. Petriello, S. Stoynev, and M. Velasco, Phys. Rev. D 88, 053003 (2013).

[12] G. Isidori, A. V. Manohar, and M. Trott, Phys. Lett. B 728, 131 (2014).

[13] B. A. Kniehl and M. Spira, Z. Phys. C 69, 77 (1995).

[14] J. Beringer et al. (Particle Data Group Collaboration), Phys. Rev. D 86, 010001 (2012).

[15] ATLAS Collaboration, Report No. ATLAS-CONF-2012-091; Report No. ATLAS-CONF-2012-109; Report No. ATLASCONF-2013-012; Report No. ATLAS-CONF-2013-013;
Report No. ATLAS-CONF-2013-015; Report No. ATLAS-CONF-2013-018; Report No. ATLAS-CONF2013-028; Report No. ATLAS-CONF-2013-030; Report No. ATLAS-CONF-2013-079; Report No. ATLAS-CONF2013-080; Report No. ATLAS-CONF-2013-095; Report No. ATLAS-CONF-2013-108; Report No. ATLAS-CONF2014-011; Eve Le Menedeu talk at Moriond 2014; G. Aad et al. (ATLAS Collaboration), Phys. Lett. B 726, 88 (2013).

[16] CMS Collaboration, Report No. CMS-PAS-HIG-12-015; Report No. CMS-PAS-HIG-13-001; Report No. CMS-PASHIG-13-002; Report No. CMS-PAS-HIG-13-004; Report No. CMS-PAS-HIG-13-012; Report No. CMS-PAS-HIG13-019; Report No. CMS-PAS-HIG-13-020; S. Chatrchyan et al. (CMS Collaboration), J. High Energy Phys. 01 (2014) 096; S. Chatrchyan et al. (CMS Collaboration), J. High Energy Phys. 05 (2014) 104; V. Khachatryan et al. (CMS Collaboration), Phys. Lett. B 736, 64 (2014).

[17] T. Aaltonen et al. (CDF Collaboration), Phys. Rev. D 88, 052013 (2013); V. M. Abazov et al. (D0 Collaboration), Phys. Rev. D 88, 052011 (2013).

[18] P. Bechtle, S. Heinemeyer, O. Stal, T. Stefaniak, and G. Weiglein, J. High Energy Phys. 11 (2014) 039.

[19] A. Falkowski, F. Riva, and A. Urbano, J. High Energy Phys. 11 (2013) 111.

[20] R. V. Harlander and W. B. Kilgore, Phys. Rev. D 68, 013001 (2003).

[21] A. D. Martin, W. J. Stirling, R. S. Thorne, and G. Watt, Eur. Phys. J. C 63, 189 (2009).

[22] R. Harnik, J. Kopp, and J. Zupan, J. High Energy Phys. 03 (2013) 026.

[23] G. Blankenburg, J. Ellis, and G. Isidori, Phys. Lett. B 712, 386 (2012).

[24] F. Goertz, Phys. Rev. Lett. 113, 261803 (2014).

[25] M. Beneke, G. Buchalla, M. Neubert, and C. T. Sachrajda, Nucl. Phys. B591, 313 (2000).

[26] P. Ball, V. M. Braun, Y. Koike, and K. Tanaka, Nucl. Phys. B529, 323 (1998).

[27] M. Beneke and T. Feldmann, Nucl. Phys. B592, 3 (2001).

[28] M. Dimou, J. Lyon, and R. Zwicky, Phys. Rev. D 87, 074008 (2013).

[29] V. M. Braun, D. Y. Ivanov, and G. P. Korchemsky, Phys. Rev. D 69, 034014 (2014).

[30] A. Khodjamirian, Lectures at the Helmholtz International Summer School "Physics of Heavy Quarks and Hadrons," July 2013, Dubna, Russia, SI-HEP-2013-15, arXiv:1312.6480.

[31] M. Beneke and J. Rohrwild, Eur. Phys. J. C 71, 1818 (2011).

[32] V. M. Braun and A. Khodjamirian, Phys. Lett. B 718, 1014 (2013).

[33] S. Aoki, Y. Aoki, C. Bernard, T. Blum, G. Colangelo, M. Della Morte, S. Drr, and A.X. El Khadra et al., Eur. Phys. J. C 74, 2890 (2014).

[34] A. L. Kagan, G. Perez, F. Petriello, Y. Soreq, S. Stoynev, and J. Zupan (to be published).

[35] G. Aad et al. (ATLAS Collaboration), J. High Energy Phys. 06 (2014) 008.

[36] (CMS Collaboration), Report No. CMS-PAS-HIG-13-034.

[37] T. Sjostrand, S. Mrenna, and P.Z. Skands, Comput. Phys. Commun. 178, 852 (2008).

[38] https://twiki.cern.ch/twiki/bin/view/LHCPhysics/CrossSections. 